\title{
Supervivencia de un catéter: un reto y un logro de enfermería
}

\author{
Isabel Crehuet Rodríguez, Pilar Méndez Briso-Montiano
}

Enfermeras del Servicio de Diálisis del Hospital Río Hortega. Valladolid

\section{Introducción}

Desde hace ya algunos años está siendo cada vez más habitual en las unidades de hemodiálisis (HD), la presencia de pacientes portadores de catéteres tunelizados ${ }^{1}$, por distintas razones que todos los profesionales de la enfermería nefrológica ya conocemos.

Desgraciadamente la red vascular no es inagotable, es más, algunos de nuestros pacientes se encuentran abocados a sucesivos fracasos de su acceso vascular y con cada uno, se van agotando poco a poco sus posibilidades de seguir dializándose, de seguir viviendo.

Existen multitud de causas que condicionan el funcionamiento del catéter; en algunas no podemos intervenir, pero en otras sabemos que jugamos un papel muy importante para su mantenimiento, funcionamiento, prevención de infecciones del propio catéter, del túnel subcutáneo, del orificio de salida, etc. Papel que va a incidir de forma muy directa en la supervivencia de los catéteres, en la morbi-mortalidad de los pacientes y en su calidad de vida.

Ante repetidas colocaciones de catéteres que resultan no funcionantes, cuando las posibilidades de poner uno nuevo que funcione se van agotando, podemos encontrarnos ante un hecho aterrador como es la última oportunidad de acceso vascular ${ }^{2-3}$. ¿Qué debe hacer la enfermería? Ser más exquisitos aún, si cabe, en el

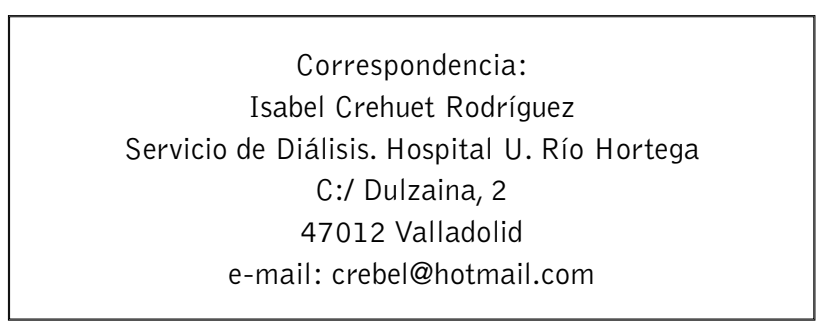

manejo de ese catéter y reinventarse día a día para que ese acceso vascular sobreviva incluso al propio paciente.

\section{Objetivos}

- Demostrar que una vena determinada, no considerada de elección sino más bien de último recurso, puede alojar un catéter perfectamente funcionante a lo largo de varios años, permitiendo una buena calidad de HD.

- Demostrar que, siguiendo escrupulosamente un protocolo o varios, como ha sido el caso a lo largo de casi 5 años, el catéter puede efectivamente pasar a ser un problema secundario en la vida del paciente, mejorando así su calidad de vida.

- Dar a conocer diferentes tipos de sellado de catéteres que también pueden ser válidos, además de los tradicionales, y que permiten mantener la permeabilidad del catéter (OSC).

- Valorar diferentes curas del orificio de salida del catéter.

- Divulgar nuestra experiencia para que pueda servir al resto de los profesionales.

\section{Paciente y método}

Paciente de 62 años, con ERC estadio 5, debida a probable pielonefritis crónica, en programa de terapia renal sustitutiva desde el año 1996.

\section{Historia Clínica}

Otros procesos que constan en ella, además de su patología renal, son:

- Dermatológicos: episodios de hidrosadenitis que cursan con abscesos que se tienen que drenar quirúrgicamente en su gran mayoría. 
- Digestivos: pancreatitis aguda, enlentecimiento del tránsito intestinal, hernia hiatal, etc.

- Reumatológicos: artritis, etc.

- Hipoacusia sensorial.

Los antecedentes de accesos vasculares y de otras técnicas de terapia renal sustitutiva antes de la implantación del catéter objeto del estudio se pueden resumir en:

- Realización de 6 fístulas arteriovenosas:

FAVI radio-cefálica izquierda.

FAVI húmero-axilar izquierda con injerto de vena safena invertida.

FAVI radio-radial derecha.

FAVI con prótesis de politetrafluoretileno (PTFE) húmero-axilar derecha.

FAVI protésica (PTFE) húmero-humeral izquierda. FAVI entre arteria femoral superficial y vena safena interna en tercio medio de muslo derecho.

- Ante la trombosis de la FAVI anterior se realiza un shunt de PTFE arterio-arterial sobre arteria subclavia derecha.

- Ingreso en programa de Diálisis Peritoneal (DP): Diálisis Peritoneal Continua Ambulatoria (DPCA) y Diálisis Peritoneal automática (DPA). Se retira a los 2 años por peritonitis fúngica, volviendo a entrar en un programa de HD. Posteriormente se volvería a intentar, dados los problemas vasculares, pero se desestimaría de nuevo por ineficacia de la técnica.

- Implantación de varios catéteres permanentes en venas yugular interna derecha, yugular interna izquierda, femoral izquierda, además de varios temporales colocados para ir resolviendo las situaciones en que se precisaba realizar HD y no se disponía de acceso vascular.

- Alarma de trasplante renal en dos ocasiones: en la primera coincidió con un episodio de fiebre por un cuadro de artritis aguda y en la segunda, las pruebas cruzadas fueron positivas, por lo que se desestimó en las dos ocasiones. Años más tarde, no se admitió su inclusión en lista de espera para trasplante por sus problemas vasculares (calcificaciones).

- Implantación de un catéter permanente en vena cava inferior. (Fig. 1)

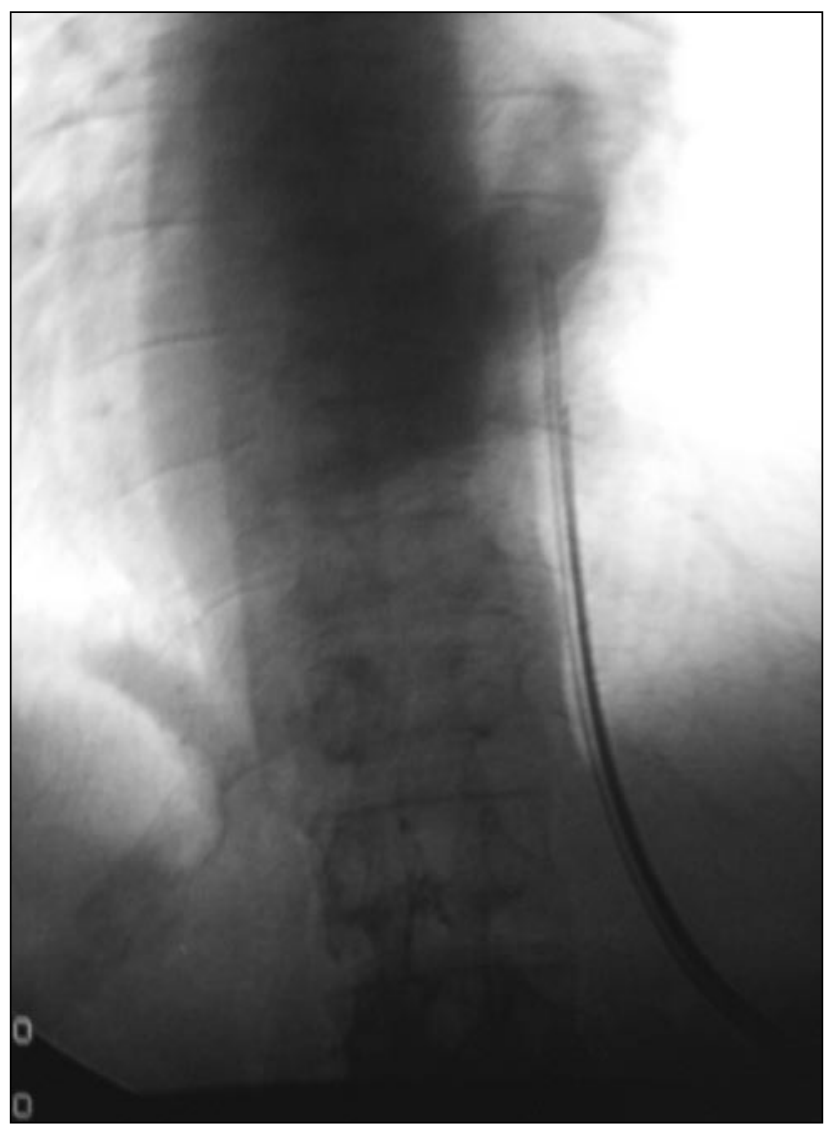

Figura 1. Rx catéter en Vena Cava Inferior

Hemos hecho un estudio retrospectivo desde la implantación de este nuevo catéter durante un periodo de aproximadamente cinco años, desde agosto del 2005 hasta Julio del 2010, que concluyó con el éxitus del paciente, debido a las complicaciones surgidas durante el post-operatorio de una intervención quirúrgica de un aneurisma en zona axilar izquierda.

Desde el momento de la implantación se empieza con tratamiento anticoagulante con acenocumarol (Sintrom ${ }^{\circledR}$ ).

A lo largo de estos cinco años hemos estudiado:

Sesiones de HD: flujo de sangre, tiempo de cada sesión, $\mathrm{Kt}$, Kt/V, presión venosa (PV)

\section{Incidencias del propio catéter}

Sellado del catéter: a lo largo de estos años hemos utilizado 4 tipos de sellado:

- $1^{\text {a. }}$ : Heparina sódica $5 \%$ y suero salino $0,9 \%$

- $2^{\circ}$ : Citrato trisódico 46,7\% (Citra-lock® 46,7\%). 
- $3^{\circ}$ : Urokinasa: $2500 \mathrm{UI} / \mathrm{ml}$

- $4^{\circ}$ : Vancomicina y Heparina sódica al 5\%: según el siguiente protocolo:

Disolver $500 \mathrm{mg}$ de Vancomicina en $25 \mathrm{ml}$ de suero salino al $0,9 \%$. De esa mezcla se cogen $2 \mathrm{ml}$ y se mezclan con $4 \mathrm{ml}$ de heparina al 5\%. De esa segunda mezcla cogemos la cantidad necesaria para sellar ambas luces del catéter, según indicación del mismo5 .

Infecciones: número, germen y resolución.

Infecciones del OSC: número, germen y evolución.

Curas del OSC: a lo largo de este proceso utilizamos 4 protocolos distintos:

- Con suero salino al 0,9\% y clorhexidina al $2 \%$.

- Con suero salino al $20 \%$.

- Con suero salino al 0,9\% y unas gotas de ciprofloxacino (cetraxal ótico).

- Con suero salino al 0,9\% y Aquacel Ag ${ }^{\circledR}$. (Fig.2).

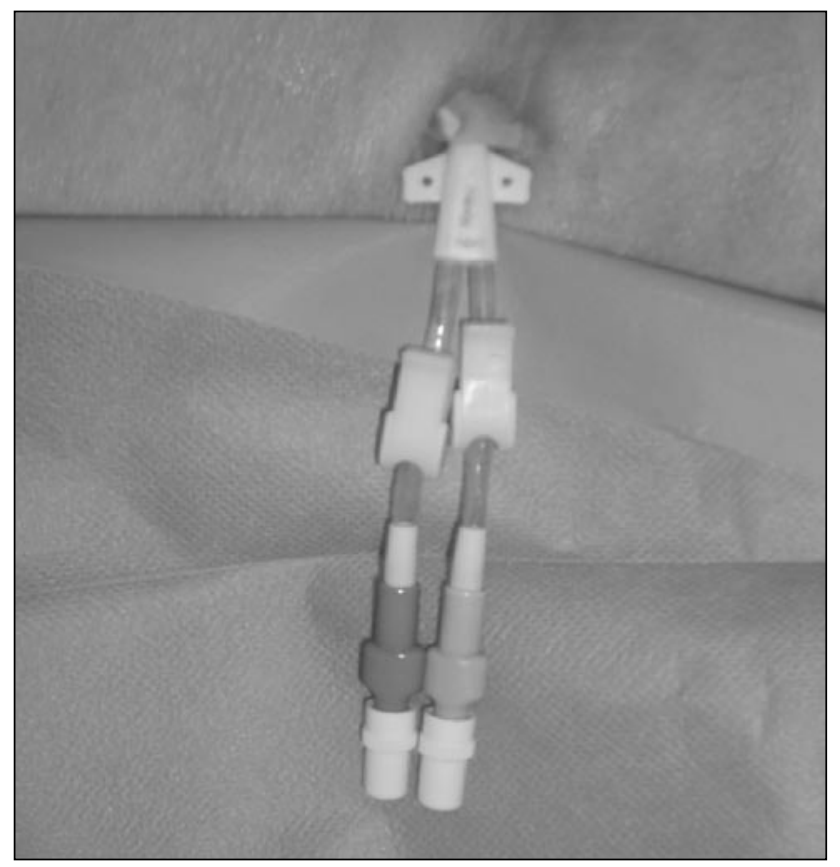

Figura 2. Cura del OSC con Aquacel $\mathrm{Ag}^{\circledR}$

Independientemente del producto utilizado para la cura del orificio, continuábamos la cura poniendo una gasa en la zona del seno cruzándola por encima del catéter para evitar úlceras de la piel por el roce de éste y acodamientos, por un lado y para evitar el pegamento de los apósitos por otro. Ponemos un apósito encima y cubrimos las ramas con un saquito.

\section{Cuidados de la piel en zona adyacente al OSC.}

Otros procesos patológicos en la vida del paciente a lo largo de estos años.

\section{Calidad de vida del paciente.}

\section{Resultados}

Sesiones de HD: la membrana del dializador utilizado a lo largo de todo este tiempo fue AN69-ST; la duración fue de $240 \mathrm{~min} / \mathrm{sesión}$, tres veces por semana; los flujos de sangre obtenidos fueron de $350 \mathrm{ml} / \mathrm{min}$ en un rango de 330-360 $\mathrm{ml} / \mathrm{min}$; la presión venosa se movió en un rango de 150-220 mm Hg, (llegó a alcanzar los 220 solamente en un episodio de infección); el Kt fue de 43, se movió en un rango de 30-48 y el Kt/V fue de 1,50 en un rango de $1,3-2,0$. (Tabla $n^{\circ} 1$ )

\begin{tabular}{|c|c|}
\hline FLUJO DE SANGRE & $350 \mathrm{ml} / \mathrm{min}(330-360 \mathrm{ml} / \mathrm{min})$ \\
\hline PRESIÓN VENOSA & $150-220 \mathrm{~mm} \mathrm{Hg}$ \\
\hline $\mathrm{Kt}$ & $43(30-48)$ \\
\hline $\mathrm{Kt} / \mathrm{V}$ & $1,50(1,30-2,00)$ \\
\hline TIEMPO DE SESIONES & 240 minutos/sesión \\
\hline
\end{tabular}

Tabla 1.

Incidencias del propio catéter: el catéter tenía una cierta tendencia a salirse, por lo que mantuvimos el punto de sutura mucho más tiempo de lo habitual. De hecho, dos meses más tarde de su inserción necesitó ser suturado a piel de nuevo, sutura que se retiró algunas semanas después, porque no cicatrizaba, y al cabo de unos días se resolvió el problema. Sin embargo, la peor incidencia que tuvimos ocurrió al cabo de dos años, a finales de agosto del 2007, cuando observamos sangrado a través del orificio de salida. Al intentar lavar la rama arterial, vimos que refluía el suero empleado, comprobando la existencia de un pequeño orificio en esa rama. Ese día y el siguiente se realizan dos sesiones a través de la rama venosa, conectando unas ramas en 
"Y", con el método de unipunción, pues el paciente se negó a la colocación de un catéter temporal para poder realizarlas. Al día siguiente se procedió al recambio del catéter por otro de iguales características a través del mismo trayecto del anterior, que presentó también un buen funcionamiento durante las sesiones de HD.

\section{Sellado del catéter:}

$1^{\text {a: }}$ : Heparina $5 \%$ y suero salino $0,9 \%$ : comenzamos sellándolo con $1 \mathrm{ml}$ de suero salino al 0,9\% más la cantidad de heparina al $5 \%$ necesaria para completar la medida del cebado en cada rama.

$2^{\circ}$ : Citrato trisódico al 46,7\%(Citra-lock ${ }^{\circledR}$ al 46,7\%): ante un episodio de infección, empezamos a sellarlo con la cantidad necesaria para llenar las ramas. Desde que se empieza a usar esta sustancia como sellante, el catéter tiene problemas de flujo, con importante disminución del Kt/V por lo que se suspendió tras cuatro sesiones y se volvió a sellar como se hacía anteriormente (heparina+ suero), resolviéndose el problema.

$3^{\circ}$ : Urokinasa: $6250 \mathrm{UI} / 2,5 \mathrm{ml}$ (2500 UI / ml). En alguna ocasión apareció un déficit de flujo arterial que nos obligó a invertir las vías, por lo que creímos conveniente utilizar esta sustancia para el sellado, en esos momentos puntuales. Fueron situaciones excepcionales y coincidieron con algún proceso infeccioso del catéter.

$4^{\circ}$ : Vancomicina y heparina al $5 \%$ según el protocolo anteriormente explicado. Se comenzó a utilizar a partir de enero de 2009 y se mantuvo hasta el final (18 meses), obteniendo buenos resultados de permeabilidad del catéter.

Infecciones: a lo largo de los dos años de supervivencia del catéter, hasta que fue recambiado, se produjeron tres infecciones causadas por Corynebacterium Striatum, que fueron tratadas con Vancomicina. En la tercera infección, ante la sospecha de que el germen estuviera alojado en el biofilm del catéter, se habló con el paciente y su esposa para volver a tratarle con Vancomicina e intentar evitar así el recambio del catéter (como estaría indicado), dados los problemas con los accesos vasculares, estando ellos de acuerdo. Posteriormente hubo que recambiarlo por la rotura, como ya se explicó anteriormente.

Tras el recambio del catéter se produjeron un total de cinco infecciones por Staphylococcus Aureus, siendo una de ellas una sepsis importante tratada con Vancomicina y Tobramicina. Se descartó la presencia de endocarditis bacteriana. El resto de las infecciones fueron tratadas con Vancomicina.

Infecciones del OSC: fueron las más numerosas. Debemos tener en cuenta que fue un paciente que además presentaba un amplio historial de procesos dermatológicos abscesos, infecciones, etc. Casi siempre había una secreción amarillenta más o menos clara. Frecuentemente se hicieron cultivos de estas secreciones, que fueron negativos incluso aún en algún caso en que coincidió con alguna infección del catéter.

En estos momentos las curas se realizaban con suero salino al 0,9\% y clorhexidina. En alguna ocasión se empleó el suero al $20 \%$ ( $\mathrm{Cl} \mathrm{Na}$ al $20 \%$ ).

Tras el recambio del catéter y hasta el final del periodo contabilizamos un total de nueve infecciones del OSC, producidas en este caso por Staphylococcus Aureus, que fueron tratadas con Vancomicina intravenosa (IV) cuando existía fiebre y hemocultivos positivos a este germen. En otras ocasiones el tratamiento fue tópico con ciprofloxacino, o con pomadas de mupirocina. Una de las veces apareció un Staphylococcus Aureus meticilin-resistente que coincidió con una aparición del mismo en el frotis nasal, siendo solamente este último resistente a mupirocina, que se trató con ácido fusídico vía nasal y el OSC con mupirocina tópica.

Otro problema que nos surgió, aunque no era una infección como tal, era la maceración de la piel que rodeaba directamente el catéter, debida a la secreción que casi siempre existía. En estos momentos decidimos curarlo con Aquacel $\mathrm{Ag}^{\circledR}$, dejándolo alrededor del catéter hasta la siguiente sesión obteniendo buenos resultados en cuanto al aspecto de la piel y la disminución de la secreción. Lo aplicamos con una cierta periodicidad, según el aspecto del OSC.

Cuidados de la piel en zonas adyacentes al OSC: se produjeron enrojecimiento, sequedad y lesiones debidas sobre todo al pegamento de los apósitos. También hay que tener en cuenta que se sujetaba el catéter con una faja abdominal que ajustaba con velcro, con lo cual siempre estaba cubierta la zona. Nuestros cuidados consistieron en aplicar vaselina en estas zonas, (Fig. 3 y 4), dejándolo sin cubrir, durante el tiempo que duraba la sesión de HD, dejándolo sin cubrir. (Figuras 
3 y 4). En algunas ocasiones aplicamos Renacare ${ }^{\circledR}$ con muy buenos resultados también e incluso en alguna ocasión llegamos a aplicar alguna pomada con corticoides cuando lo anterior no era suficiente. Hicimos siempre mucho hincapié en que se aplicara crema en todo el cuerpo cuando se aseara, pues tenía mucha sequedad de piel.

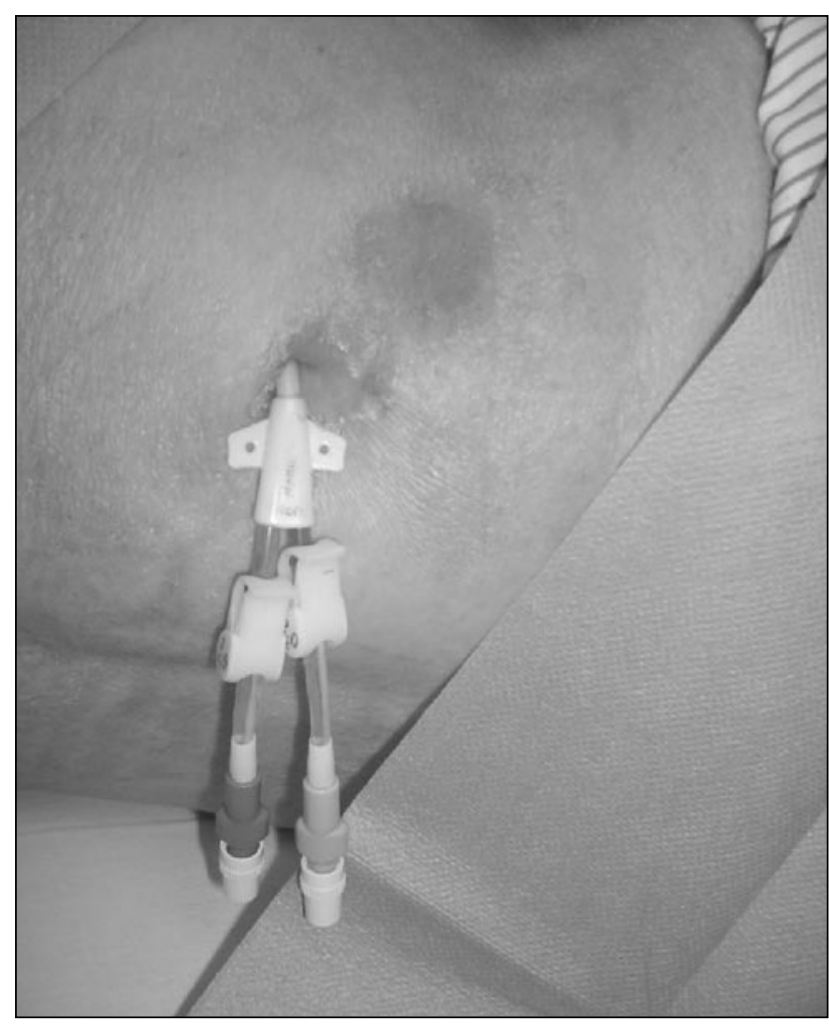

Figura 3. Lesiones en OSC y zonas adyacentes

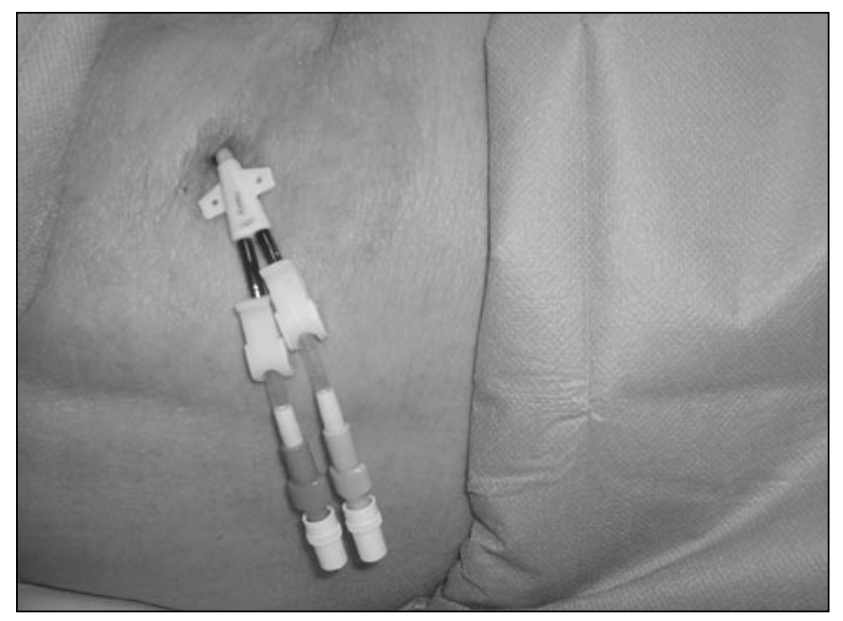

Figura 4. Aspecto del OSC tras las curas, previo a la siguiente HD

\section{Otros procesos en la vida del paciente durante este} tiempo:

- Retirada quirúrgica de la prótesis húmero-cefálica izquierda por infección por Proteus Mirabilis.

- Intervención quirúrgica para recambio de válvulas cardiacas aórtica y mitral, de la que se recuperó bastante bien.

- Episodios de hidrosadenitis con abscesos en distintas zonas que fueron drenados quirúrgicamente.

- Procesos reumatológicos: monoartritis aguda de rodilla izquierda, quiste poplíteo,etc.

- Procesos digestivos con importante enlentecimiento del tránsito digestivo.

Calidad de vida: a pesar de todo lo descrito anteriormente, su calidad de vida mejoró considerablemente, valga como prueba que fue la única vez que se atrevió a irse de vacaciones, lejos de su hospital de referencia, y algunos fines de semana a su pueblo (a $100 \mathrm{Km}$ de dicho hospital). El paciente mejoró psicológicamente, manifestaba encontrarse con mejor ánimo y bastante cómodo con este catéter, pues le permitía hacer una vida mucho más normal de la que había tenido hasta ahora.

\section{Discusión}

Desde el momento en que tuvimos este caso en nuestras manos, nuestro objetivo fue cuidar este catéter para alargar su supervivencia todo el tiempo posible. Aplicamos el protocolo de conexión y desconexión de HD que utilizábamos en la unidad, de forma muy rigurosa y a pesar de ello no pudimos evitar las infecciones que se iban produciendo. Fuimos aplicando distintos protocolos en cuanto al sellado $0^{4-5}$ cuando empezamos a ver el más mínimo problema. En cuanto a las curas del OSC, muchas veces nos basamos en nuestra experiencia en otro tipo de curas, para aplicarlo a éstas, casi de una manera intuitiva, como nos ocurrió con el Aquacel $\mathrm{Ag}^{\circledR}$, pues no encontrábamos muchos casos en la literatura que pudieran informarnos de casos semejantes. Pensamos que tenía mucho que ver, además de las propias características del paciente, la zona donde estaba implantado el catéter, pues no nos ocurría con otros catéteres aunque estuvieran implantados en venas femorales, que son más sensibles a las infecciones. 
A medida que el tiempo pasó y fuimos sorteando las complicaciones, empezamos a considerar casi una obligación divulgar nuestra experiencia entre nuestros colegas. También nos animó que cuando fue de vacaciones y se dializó en otro centro, la información que adjuntamos al informe médico sobre los cuidados de este catéter sirvió de gran ayuda, según nos manifestaron las compañeras de dicho centro, pues no habían visto antes un catéter en esa zona. Esperamos que este estudio pueda ayudar a otros compañeros a lo largo de su vida profesional, ya que nuestro objetivo como profesionales de enfermería, es conseguir la excelencia en los cuidados para mejorar la calidad de vida de los pacientes.

\section{Conclusiones}

Podemos decir, que al menos en este caso, la vena cava inferior fue un buen acceso vascular para conseguir una hemodiálisis eficaz durante mucho tiempo; que aplicando los distintos protocolos ya citados, fuimos sorteando todas las complicaciones y conseguimos mantener ese catéter funcionante hasta el final, mejorando considerablemente la calidad de vida del paciente.

\section{Agradecimientos}

Al paciente y a su esposa, principal cuidadora y muy colaboradora, por la confianza que depositaron en nosotros.

A todo el personal que colaboró en esta ardua tarea a lo largo de estos años.

\section{Bibliografía}

1. Guías de acceso vascular en hemodiálisis. Nefrología 2005; 25 (sup. 1): 1-174.

2. I.Crehuet, S. Mendinueta, P. Méndez, R. González. Catéter translumbar en vena cava inferior: última opción de acceso vascular para hemodiálisis. Rev. Soc. Esp. Enferm. Nefrol. 2008; 11 (3): 238/241 11.

3. Lung. GB, Trerotola S0, Scheel PJ Jr. Percutaneus translumbar inferior vena cava cannulation for hemodialysis. Am J Kidney Dis 1995. 25 (5), 732-737.

4. A. Baltrons, S. Coll Vinyoles, C. Font Senen, M. Jubany López,C. Plana Illa, A. Sunyer-García. Estudio comparativo del sellado de catéteres con citrato trisódico o heparina sódica más gentamicina. Rev. Soc. Esp. Enferm. Nefrol. 2008; 11 (2): 95-100.

5. J.M. Campistol, A. Darnell. Protocolos del Servicio de Nefrología y Trasplante Renal 2008. Hospital Universitario Clínico de Barcelona. Pag.296-297. 